\title{
Professionalität und Postkonventionalität. Entwicklungstheoretische und soziologische Perspektiven auf professionalisiertes Handeln
}

Boris Zizek (Universität Mainz)

\section{Einleitung}

Im Folgenden geht es mir erstens darum, die zentrale Stellung postkonventionellen Moralurteils im professionalisierten Handeln aufzuzeigen, es anhand einiger Fallbeispiele als Voraussetzung vollgültiger Professionalisierung herauszuarbeiten.

Mit dem Begriff des professionalisierten Handelns wird an Ulrich Oevermanns soziologische Professionalisierungstheorie angeknüpft, die für sich in Anspruch nimmt, Professionen nicht nur wie die klassische Theorie in ihren „institutionellen Erscheinungsformen der relativen Autonomie“ (Oevermann 1996, 70) zu erfassen, sondern als Antwort auf spezifische Handlungsprobleme. Die systematische Verortung und Bestimmung professionalisierten Handelns wird an späterer Stelle vorgenommen. Oevermann unterscheidet zwei Ebenen professionalisierten Handelns. Die erste sind „,...] die Bereiche professionalisierten Handelns im Namen von Erkenntniskritik, das heißt von Wissenschaft und Kunst" (ebd., 82). Der zweite, eine doppelte Professionalisierung beinhaltende Bereich

\footnotetext{
* Vorliegender Aufsatz ist ein überarbeiteter Vortrag, den ich auf der Jahrestagung „Beruf und Moral" 2011 des Arbeitskreises Moral in Salzburg halten durfte. Ich danke für die ausführliche Diskussion insbesondere Klaus Beck, Georg Lind und Fritz Oser.
} 
umfasst die intervenierenden Formen professionalisierten Handelns, Therapie, Rechtspflege, Architektur und pädagogisches Handeln.

Anhand Johann Wolfgang Goethes 1774 erschienenen „Die Leiden des jungen Werthers" (Goethe 1994) werde ich zunächst exemplarisch Stufen des Prozesses einer Professionalisierung auf der ersten Ebene professionalisierten Handelns aufzeigen. In diesem ersten Schritt wird professionalisiertes Handeln mit Lawrence Kohlberg, Robert Kegan und Detlef Garz aus der Perspektive stufenförmiger Entwicklung untersucht und die zentrale Stellung postkonventionellen Moralurteils herausgearbeitet.

In einem zweiten Schritt wird aufgezeigt, dass in Oevermanns theoretischer Skizze einer revidierten Professionalisierungstheorie Postkonventionalität eine unausgesprochene Voraussetzung professionalisierten Handelns darstellt. In einem dritten Schritt wird schließlich, ausgehend von Kegans Fallbeispiel eines postkonventionellen israelischen Militärarztes, postkonventionelles Moralurteil in Oevermanns professionalisierungstheoretischer Studie zu Arthur Schnitzlers Drama „Professor Bernhardi“ (Oevermann 1997) aufgewiesen. Bezeichnenderweise ist der Professionalisierte darin mit einer Situation konfrontiert, in der er sich, um professionalisiert handeln zu können, gegen herrschende Konventionen durchsetzen muss.

\section{Stufen des Professionalisierungsprozesses in Johann Wolfgang Goethes Die Leiden des jungen Werthers}

Um nun ,[...] professionalisiertes Handeln im Namen von Erkenntniskritik“ (Oevermann 1996, 82) im Kontrast zu Alltagshaltungen und Vorformen professionalisierten Handelns in den Blick zu nehmen und den Stellenwert postkonventionellen Moralurteils darin kenntlich zu machen, beginne ich mit einer verdichteten, fokussierten Darstellung einer Sequenzanalyse des Anfangs des ersten Briefes Werthers an seinen besten Freund Wilhelm und werde im Anschluss auf die vorangehende Vorrede des fiktiven Herausgebers eingehen ${ }^{1}$. Die zusätzliche Rahmung bzw. die darin zum Ausdruck kommende Haltung des Herausgebers stellt nämlich im Vergleich zu Werther einen Entwicklungsschritt dar, der professionalisiertes Handeln ermöglicht.

\footnotetext{
${ }^{1}$ Eine ausführliche Sequenzanalyse liegt in meiner Dissertation „Probleme und Formationen des modernen Subjekts- Zu einer Theorie universaler Bezogenheiten des Subjekts“ vor, die 2011 erscheint.
} 
"Am 4. Mai“

Absenz einer Anrede

Zwei Besonderheiten dieser Brieferöffnung möchte ich hervorheben. Mit der Tilgung der Angabe der Jahreszahl in der Datierung des Briefes wird die Orientierung an mit der Moderne sich breitenwirksam durchsetzenden antizyklischen, gesellschaftlichen Rhythmen abgebaut, die Fossier als den Beginn der Zeit der Kaufleute bezeichnet hat.

Die Tilgung der üblichen Anrede, die im Sturm und Drang nicht selten war (Nickisch 1991) und eine permanente Präsenz und Verfügbarkeit des Gegenübers impliziert, erzeugt im positiven Falle eine Kommunikationsform intimen, begleitenden und bestärkenden Austauschs. Beide Tilgungen setzen sich gegen etablierte Konventionen durch.

Wie froh bin ich, daß ich weg bin! Bester Freund, was ist das Herz des Menschen, Dich zu verlassen, den ich so liebe, von dem ich unzertrennlich war und froh zu seyn!

Sofort ins Auge fällt der initiale Ausruf der Erleichterung über den erfolgten Rückzug aus dem Bereich der Verfügbarkeit. Die gesamte Eröffnungssequenz zeichnet sich durch Expressivität und Spontaneität der Äußerung aus.

Die Sequenz „was ist das Herz des Menschen“ artikuliert das Problematischgewordensein einer zentralen, empathischen Instanz der Entschließung, dem Herz des Menschen. Im Allgemeinheitsgrad der Fragestellung manifestiert sich eine philosophische Haltung, in der die Alltagsroutinen einer Authentizitätsprüfung unterzogen werden. Im Folgenden wird ein beglückendes, obwohl aus Bindungen lösendes Autonomiestreben skandalisiert, was dessen Neuund Fremdheit indiziert.

[...] Die arme Leonore! Und doch war ich unschuldig. Konnt' ich dafür, daß, während die eigensinnigen Reize ihrer Schwester mir eine angenehme Unterhaltung verschafften, daß eine Leidenschaft in dem armen Herzen sich bildete? Und doch - bin ich ganz unschuldig? Hab`ich nicht ihre Empfindungen genährt? hab` ich mich nicht an den ganz wahren Ausdrücken der Natur, die uns so oft zu lachen machten, so wenig lächerlich sie waren, selbst ergetzt, hab` ich nicht - O was ist der Mensch, daß er über sich klagen darf!

Diese wenig später einsetzende Auseinandersetzung bringt ein akutes, Werthers Leiden verursachendes Ringen zweier Perspektiven zum Vorschein, die es nun zu differenzieren gilt.

Mit dem Ausruf "Die arme Leonore“ wird das Individuum in seiner biographischen Eingeschränktheit empathisch und exkulpierend in den Blick genommen.

Dass Werther auch in der Ferne die Schuldfrage gegen sich richtet, ist ein Hinweis auf die erlangte Kompetenz moralischen Urteilens der Stufe 4. Diese Moralstufe manifestiert sich auch in der im Folgenden erörterten Frage des 
Schuldiggewordenseins aufgrund frühzeitiger Erhitzung der Jugend, in der das Motiv der Aufrechterhaltung einer Ordnung zum Ausdruck kommt. Die hier operierende Perspektive kann man in ihrer Ordnungsbesorgtheit soziologisch mit Kegan als Perspektive der Gruppe oder mit Garz als Systemperspektive begreifen.

Stufe 4 konzentriert sich auf das moralische Verhältnis zum umfassenden sozialen System. Gesetze und ihre Einhaltung sowie generell ein bewusstes Verhältnis zur sozialen Ordnung bilden den zentralen Punkt dieser Orientierung. (Garz 1996, 59)

Im Gegensatz zur Systemperspektive zeichnet sich der oben bereits thematische biographische, empathische Blick, den man als ein Moment einer Perspektive des Individuums fassen kann, auch durch eine ästhetische Öffnung für die authentischen Regungen des Individuums aus, die Werther als "ganz wahre Ausdrücke der Natur" thematisiert.

In der Systemperspektive hingegen werden die Regungen der Jugend summarisch, subsumtionslogisch ironisiert, nicht ernst genommen und mit der Legitimation der Ordnungserhaltung nicht anerkannt.

Mit Kegan kann das Ringen der Systemperspektive und der Perspektive des Individuums als Übergang von der konventionellen zur post-konventionellen Ebene des moralischen Urteils, als Stufe $4 \frac{1}{2} 2$ bestimmt werden (Kegan 2008, 308).

[...] Ich will, lieber Freund ich verspreche dir`s ich will mich bessern, will nicht mehr ein bisschen Übel, das uns das Schicksal vorlegt, wiederkäuen, wie ich`s immer getan habe, ich will das Gegenwärtige genießen, und das Vergangene soll mit vergangen seyn.

Der beste Freund wird hier als Vertreter der Systemperspektive eingeführt. Werther verspricht ihm, die Perspektive des Individuums, die er hier in Selbstagitation zu bloßer Grübelei entwertet, aufzugeben.

Der beste Freund versteht nicht, was Werther, der sich einerseits empathisch den Eingeschränktheiten und authentischen Regungen der Individuen öffnet und sich andererseits für diese ordnungszersetzende Praktik durch die internalisierte Systemperspektive anklagt, eigentlich umtreibt, was die Ursache seiner Leiden ist. Hier kommt die strukturelle Einsamkeit zum Ausdruck, die Garz bezüglich postkonventionell Urteilender festgestellt hat (Weber 1981, 1).

Die Lösung von Werthers Leiden ist aus der Sicht einer Haltung, der die Stufe 4 moralischen Urteilens zugrunde liegt, die Spannung aus der Perspektive des Individuums herauszunehmen, sie gleichsam einzufrieren.

Nun soll die Vorrede und darin die Haltung des Herausgebers in den Blick genommen werden.

Was ich von der Geschichte des armen Werther nur habe auffinden können, habe ich mit Fleiß gesammelt, und lege es euch hier vor, und weiß, daß ihr mir`s danken werdet. Ihr 
könnt seinem Geiste und seinem Charakter eure Bewunderung und Liebe, seinem Schicksal eure Tränen nicht versagen.

Und du gute Seele, die du eben den Drang fühlst wie er, schöpfe Trost aus seinem Leiden, und laß das Büchlein deinen Freund seyn, wenn du aus Geschick oder eigener Schuld keinen nähern finden kannst.

Mit der angekündigten Textsammlung zur "Geschichte des armen Werther" nähert sich der Herausgeber Werther von einer biographisch empathischen Perspektive aus, die auch schon bei diesem selbst festgestellt wurde.

Aus der angekündigten Vorrede ist zu entnehmen, dass der Herausgeber eine uninterpretierte $(, \ldots$ mit Fleiß gesammelt, ...“), biographische Textsammlung eines Gescheiterten („Was ich von der Geschichte des armen Werther nur habe auffinden können, ...") angefertigt hat, welche er durch eine Vorrede gerahmt herausgibt (,,$\ldots$ und lege es euch hier vor, ...").

Untersucht man diese Handlung zunächst ganz unabhängig von der möglichen Intention, dem subjektiv gemeinten Sinn nach Max Weber ${ }^{2}$, mit dem der Handelnde sie vielleicht versehen würde, dann ist festzuhalten, dass sie Einblick in Dokumente verschafft, die erstens im Sinne objektiver, uninterpretierter Daten Auskunft über biographische Ereignisse (Geburt, Heirat, Migration, Umzug...) verschaffen. Darüber hinaus geben solche Dokumente in der Regel auch Auskunft über die subjektive Beurteilung und Aneignung dieser Ereignisse, gleichsam die lyrische Seite der Äußerung.

Versteht man Empathie als Orientierung und Interesse für die subjektiven Beweggründe eines Handelns, dann stellt die Anfertigung einer biographischen Dokumentensammlung die Vorbereitungsphase einer gesteigerten, den biographischen Erfahrungsraum eines Individuums berücksichtigenden, empathischen Handlung dar, gleichsam eine performative Empathie (Garz 1996, 66). Eine biographische Dokumentensammlung ermöglicht es, das Handeln vor dem Hintergrund des Erfahrungsraumes zu verstehen.

Nun kann es sich bei der biographischen Dokumentensammlung um eine Handlung etwa innerhalb einer detektivischen, wissenschaftlichen, belletristischen, betrügerischen oder biographischen Gesamthandlung handeln. Wichtig ist, festzuhalten, dass der empathische Charakter der Handlung von diesen möglichen strategischen Rahmungen in einer gewissen Hinsicht unberührt bleibt. Auch eine betrügerische Praxis, für die es hier, wie wir sehen werden, keinerlei Hinweis gibt, muss, um erfolgreich zu sein, die angefertigte Dokumentensammlung anschließend

\footnotetext{
${ }^{2} \mathrm{Zu}$ diesem Begriff hat mich Georg Lind auf der in Fußnote (S. 97) genannten Tagung angeregt.
} 
biographisch empathisch in den Blick nehmen, um sich durch das Verstehen des Opfers einen Vorteil zu verschaffen. Gleichwohl handelte es sich insbesondere in diesem Fall um eine gleichsam verunreinigte Empathie, insofern die Empathie nicht Grundlage eines Mitleids oder einer Zuwendung ist, sondern im Gegenteil zur Erhöhung des Leidens beiträgt.

Welche Informationen bezüglich der motivationalen Einbettung der empathische Beschäftigung vorbereitenden und damit faktisch empathischen Handlung stehen dem Interpreten des Werther noch zur Verfügung?

Der Umstand, dass es sich um eine herausgegebene Textsammlung handelt, die Attribuierung Werthers als „arm“ und die geäußerte „Bewunderung“ sprechen aufgrund der explizit mitleidigen und bewundernden Haltung Werther gegenüber gegen eine betrügerische, detektivische und wohl auch gegen eine wissenschaftliche Gesamthandlung.

Werther wird als ein tragischer Held eingeführt, der trotz bester Eigenschaften scheitert. Es wird hier also nicht bloß wie durch Werthers besten Freund eine Einfrierung der Perspektive des Individuums als Lösung der Problemsituation vorgeschlagen. Die hohe Wertschätzung Werthers durch den Herausgeber erlaubt nur eine neuartige Integration.

Der Umstand, dass der Herausgeber die Leser duzt, schließt einen belletristischen Kontext aus, in dem man sich an ein anonymes Publikum wendet, das in aller Regel gesiezt wird.

Das Duzen impliziert eine Vergemeinschaftung, die in einer direkten Bekanntschaft oder einer gemeinsamen kritischen Lage wurzeln kann. Die Anrede einer nicht persönlich bekannten „guten Seele“ spricht für die zweite Lesart. Dieser Leidensgemeinschaft präsentiert der Herausgeber hier einerseits einen bewunderungswürdigen Fall, der ihre Krise exemplarisch zum Ausdruck bringt, andererseits spricht er aber im Gegenzug auch den möglichen Eigenanteil an der Krise an („eigener Schuld“). Die Gleichzeitigkeit von fallspezifischer Anerkennung und Thematisierung von möglichen Eigenanteilen bringt eine therapeutische Haltung zum Ausdruck. Der Ausschluss betrügerischer, belletristischer, detektivischer motivationaler Einbettungen und die Feststellung einer gleichsam therapeutischen Haltung in der Rahmung der Dokumentensammlung, spricht für eine besonders reine Form der Empathie, insofern die empathische Praxis nicht strategisch, mit sekundären Motiven in den Dienst der Vorteilsgewinnung gestellt wird, sondern, dabei nicht im Mitleid stecken bleibend, dem Wohl einer Gemeinschaft dient, die in der Krise ist. Insofern nicht einmal Hinweise auf eine Entlohnung für die therapeutische Intervention vorliegen, scheint es sich hier sogar 
um ein Beispiel besonders unstrategischen, von sekundären Motiven nicht entstellten Handelns zu handeln.

Kontrastiert man nun die drei Protagonisten bester Freund, Werther und den Herausgeber, so kann man sie, analog ihrer Moralentwicklung von Stufe 4 zu 4 1/2 und 5, als Stufen eines Professionalisierungsprozesses in den Blick nehmen:

Werthers bester Freund, ein Vertreter der Systemperspektive und damit der Stufe 4 des moralischen Urteils zeigt eine adultistische Haltung, die sich an der Erhaltung der sozialen Ordnung orientiert und als Umgang mit adoleszenter Haltung eine diese abschaffende Einfrierung der empathischen, Konventionen problematisierenden Perspektive des Individuums vertritt.

Werther selbst, dessen Krise als eine des Übergangs von der konventionellen zur post-konventionellen Ebene bestimmt wurde, macht nun die Perspektive des Individuums gegen die des Systems stark, so dass es zu jenem charakteristischen Ringen der Perspektiven kommt. Seine Wahrnehmung des Gegenübers zeichnet sich durch Empathie für die biographische Lage des Individuums und eine unvoreingenommene Öffnung für die Äußerungen des Individuums aus.

Der Herausgeber zeigt nun einerseits wie Werther selbst eine biographische, empathische, sich dem Individuum in seinem konkreten Ausdruck öffnende Haltung. Er bringt auch seine Zustimmung für diese Seite Werthers zum Ausdruck. Im Gegensatz zur adultistischen Haltung des besten Freundes propagiert er also keine Einfrierungsstrategie dieser adoleszenten, sturm- und dränglerischen Perspektive Werthers. In Differenz zu Werther jedoch vermag er aber auch den Eigenanteil und damit die Perspektive der Gruppe bzw. des Systems in seine Betrachtung zu integrieren. Neben dem Geschick etwa nennt der Herausgeber auch die „eigene Schuld“ als eine mögliche Ursache der Vereinsamung der "guten Seele“.

Der Herausgeber vermag sich im Gegensatz zu Werther aus der Versenkung in eine besondere Situation wie der Lebensgeschichte eines Scheiternden zu lösen. Durch diese kühle, Distanz und Abwägung ermöglichende Seite gelingt ihm mit der biographischen Textsammlung ein geschlossenes Werk. Aufgrund dieser gelungenen Integration der Perspektive des Individuums und des Systems bzw. der Gruppe ist der Herausgeber der Stufe 5 der Moralentwicklung zuzuordnen. Postkonventionalität erlaubt also einerseits eine fallspezifische, falsche adultistische Abwehr vermeidende Einlassung auf die besondere biographische Lage eines Individuums, andererseits eröffnet sie eine Berücksichtigung der System- bzw. Gruppenperspektive, wodurch eine relative Distanz gewonnen wird, die im Gegensatz zu Werthers heroischem Festhalten an der Perspektive des Individuums das Gelingen eines Werkes und die eigene Unversehrtheit ermöglicht. Hier kann also von einer Koexistenz oder Integration von kritischer, Authentizität prüfender, 
biographisch-empathischer Perspektive des Individuums und Eigenanteile an einer problematischen Situation berücksichtigender, Werkgestaltung und eigene Unversehrtheit ermöglichender Distanznahme von individueller Betroffenheit einer Systemperspektive gesprochen werden.

Mit Kegan können die Einseitigkeiten der gleichsam vorprofessionellen Moralstufen 4 und 4 1/2 nochmals zusammengefasst werden.

\begin{abstract}
Auf Kohlbergs Stufe 4 wurde der alte Konflikt zwischen Individuum und Gruppe entschieden (ohne überhaupt einen Begriff des Individuums zu konstruieren). Die Stufe, die wir jetzt betrachten, Stufe $4 \frac{1}{2}$ im Kohlbergschen Modell, ist eine Übergangsphase, in der die Entscheidung ganz zugunsten des Individuums ausfällt [...] (Kegan 2008, 100).
\end{abstract}

\title{
3. Moralische Postkonventionalität in Ulrich Oevermanns Professionalisierungstheorie
}

Nachdem professionalisiertes Handeln aus entwicklungstheoretischer Perspektive näher bestimmt worden ist, soll nun auf Grundlage einer Skizze der soziologischen Professionalisierungstheorie Oevermanns der Stellenwert aufgezeigt werden, den darin Postkonventionalität einnimmt.

In Oevermanns Professionalisierungstheorie wird professionalisiertes Handeln zunächst anhand dreier Ableitungsbasen verortet. Anschließend wird professionalisiertes Handeln der ersten Ebene, das in einem erfahrungswissenschaftlichen Habitus kumuliert, von einem interventionspraktischen professionalisierten Handeln unterschieden und hinsichtlich des Gegenstandsbereiches differenziert.

1. Auf der ersten Ableitungsbasis wird professionalisiertes Handeln als der gesellschaftliche Ort der Vermittlung der durch methodisches und praktisches Verstehen entstehenden Differenz von Theorie und Praxis begriffen. Diese Vermittlung habe in Respektierung und mit dem Ziel der Wiederherstellung der beschädigten Autonomie der Praxis zu geschehen, da wissenschaftliche Erkenntnis das praktische Entscheiden in eine offene Zukunft hinein nicht ersetzen könne (Oevermann 1996, 79).

2. Auf einer zweiten Ableitungsbasis, auf der die beiden humanspezifischen Lebensvollzugsphasen Krisenbewältigung und Routineexekution unterschieden werden, wird professionalisiertes Handeln von unternehmerischem Handeln und Intellektuellen-Räsonement noch ungeschieden, doch in Abgrenzung zum bürokratischen Handeln „,[...] dem Komplex der systematischen Erneuerung durch Krisenbewältigung" (ebd., 
82) zugerechnet. Dieser Grad der Differenzierung durch spezialisierte Instanzen vollzieht sich erst im Übergang zur Moderne (vgl. ebd., 81).

3. Auf der dritten Ableitungsstufe wird durch eine Differenzierung von „zwei Phasen [...] der Krisenbewältigung“ (ebd., 82), einer „primären Phase der aktiv-praktischen Entscheidung zu einer Aktion“ (ebd., 82) und einer zweiten Phase der "Rekonstruktion dieser spontanen, selbstcharismatisierten ersten Entschließung" (ebd., 83) professionalisiertes Handeln der zweiten, also der Rekonstruktionsphase der Krisenbewältigung zugerechnet.

Professionalisiertes Handeln erfordere daher einerseits eine Neutralisierung der praktischen Charismatisierung, welche die Instanzen der Entscheidungsphase der Krisenbewältigung benötigen, andererseits sollte es dadurch wiederum auch nicht "auf die Seite des bürokratischen Routinehandelns" (ebd., 85) umspringen. Oevermann fasst die so entstehende Konstellation „,...] als eine widersprüchliche Einheit von ganzer Person und unpersönlicher Rollenförmigkeit, von diffuser und spezifischer Sozialbeziehung" (ebd., 86).

In Oevermanns Verortung professionalisierten Handelns in der Rekonstruktionsphase der Lebensvollzugsphase Krisenbewältigung wird in dem relativierenden, geltungsüberprüfenden Moment dieses Handelns dessen grundsätzlich postkonventioneller, mit vorhandenen Routinen kritisch brechender Charakter deutlich.

Eine weitere Differenzierung professionalisierten Handelns findet über den Gegenstandsbereich möglicher Geltungsüberprüfung, die „materialen Lebensbereiche, die [...] in eine [...] Geltungskrise geraten können“ (ebd., 89), statt. Die ersten beiden Problemfoci sind die „Aufrechterhaltung und Gewährleistung einer kollektiven Praxis von Recht und Gerechtigkeit im Sinne eines die jeweils konkrete Vergemeinschaftung konstituierenden Entwurfs“ (ebd., 88) und „[...] die Aufrechterhaltung und Gewährleistung von leiblicher und psychosozialer Integrität des einzelnen im Sinne eines geltenden Entwurfs der Würde des Menschen“ (ebd., S. 88). Die beiden, genannten Problemfoci „[...] stehen ihrerseits in einem polaren Spannungsverhältnis zueinander, analog der dialektischen widersprüchlichen Einheit von Individuum und Gesellschaft" (ebd., 88).

Ich möchte darauf hinweisen, dass dieses Spannungsverhältnis der Problemfoci professionalisierten Handelns, die „[...] dialektische widersprüchliche Einheit von Individuum und Gesellschaft" (ebd., 88), oben, in Werthers erstem Brief, als das Ringen der Perspektiven des Individuums und des Systems festgestellt worden ist. 
Oevermann führt einen weiteren Fokus ein, der sich verschärfend auf das thematische Spannungsverhältnis der Foci Individuum und Gesellschaft auswirkt.

Diese dabei zu leistende Vermittlungsarbeit steigert sich in dem Maße, in dem die beiden gegensätzlichen Foci $[\ldots]$ zum Gegenstand eigenlogischer Geltungsüberprüfung warden (ebd., 92).

Auch diese Steigerung der Vermittlungsarbeit liegt bei Werther vor, der sich mit dem Ausruf „Wie froh bin ich, dass ich weg bin!“ ganz im Sinne der Verortung professionalisierten Handelns in der Rekonstruktionsphase aus dem Alltagskontext zurückzieht, um dem Ringen der beiden in Spannung stehenden Perspektiven einen größeren Raum zu verschaffen, die Alltagsroutinen vor diesem Hintergrund einer philosophischen, auf allgemeine Zusammenhänge ausgerichteten Authentizitätsprüfung zu unterziehen.

Erst dem Herausgeber gelingt es die aus der Verortung professionalisierten Handelns in der Rekonstruktionsphase der Lebensvollzugsphase Krisenbewältigung hervorgehende Forderung der Koexistenz von Einlassung als ganzer Mensch und Neutralisierung praktischer Charismatisierung zu realisieren. Der professionalisierte Künstler kann mithilfe der im Werther gestalteten Einbettungsverhältnisse im Sinne der „[...] widersprüchlichen Einheit von ganzer Person und unpersönlicher Rollenförmigkeit, von diffuser und spezifischer Sozialbeziehung“ (ebd., 86) als der distanzierte Herausgeber seiner Selbst als eines als ganzer Mensch Involvierten gefasst werden. Charles Baudelaire scheint genau diese Haltung, die dem Herausgeber im Gegensatz zu Werther das Gelingen eines Erfahrungen vermittelnden Werkes ermöglicht, an Eugène Delacroix fasziniert zu haben.

Delacroix ist die Verkörperung des Genies, insofern als er leidenschaftlich in die Leidenschaft verliebt ist und zugleich ganz kalt die geeigneten Mittel sucht [...] (Fischer 1996, 658).

Die „,...] widersprüchliche Einheit von ganzer Person und unpersönlicher Rollenförmigkeit, von diffuser und spezifischer Sozialbeziehung“ (Overmann 1996, 86) im professionalisierten Handeln eröffnet eine geltungsüberprüfende Vermittlung der Perspektiven des Individuums und der Gesellschaft, die einerseits die Autonomie der Praxis respektiert und andererseits auch die eigene Unversehrtheit und das Gelingen der Werkgestaltung gewährleistet.

Dies entspricht der gelungenen Integration der beiden Perspektiven, die oben aus entwicklungstheoretischer Perspektive mit der Moralstufe 5 gefasst wurde. 


\section{Moralische Postkonventionalität in intervenierendem, professionalisiertem Handeln}

Kegan veranschaulicht in seinen Entwicklungsstufen des Selbst ganz im Sinne der vorliegenden Argumentation postkonventionelles Moralurteil mit dem Fallbeispiel eines Arztes, der für das israelische Militärarzt arbeitet und „[...] der, im Unterschied zu den meisten seiner Kameraden, verwundeten Arabern und Israelis die gleiche ärztliche Behandlung zukommen ließ [...] Trotz der unverhehlbaren Zuneigung, die wir für Angehörige der eigenen Gruppe (die Teil unserer $>$ Form $<$ ist) spüren, wird unsere Gruppenidentifikation nicht zum letzten kontrollierenden Prinzip“ (Kegan 2008, 301). Die Überschreitung der System- bzw. Gruppenperspektive, die der eigenen Gruppe eine Präferenz zukommen lässt, ermöglicht es hier dem Militärarzt, dem aus einer anderen Gruppe stammenden Patienten eine fallspezifische Zuwendung zukommen zu lassen.

Die therapeutische Praxis, die in Kegans "Metatheorie der Therapie" thematisch ist und die von Oevermann dem Problemfokus der Erhaltung "leiblicher und psychosozialer Integrität des einzelnen“ (Oevermann 1996, 88) zugerechnet wird, stellt gegenüber der bisher thematischen systematischen Geltungsüberprüfung eine doppelte Professionalisierung dar, insofern sie sowohl in den erfahrungswissenschaftlichen Habitus einsozialisiert ist als auch temporär Entscheidungen für die in die Krise geratene Praxis trifft (ebd., 124).

Nachdem die zentrale Stellung postkonventionellen Moralurteils in Oevermanns theoretischer Skizze aufgezeigt wurde, soll nun exemplarisch an einer Fallstudie Oevermanns die Bedeutsamkeit kenntlich gemacht werden, die Postkonventionalität in Oevermanns professionalisierungstheoretischem Denken unausgesprochen einnimmt.

In einer literatursoziologischen Analyse Arthur Schnitzlers Dramas „Professor Bernhardi“ rekonstruiert Oevermann anhand des Klinikchef Bernhardi, in dem nach Oevermann exemplarisch die Professionsethik eines Sigmund Freud gestaltet ist, ein theoretisches Modell interventionspraktischen, professionalisierten Handelns (Oevermann 1997).

Den Hintergrund des Dramas bildet das Wien der Jahrhundertwende der k. u. k. Monarchie. Es gehört in diesem Kontext, wie in der zentralen Szene des Dramas deutlich wird, zur Konvention, dass bei absehbarem Versterben eines Patienten ein Pfarrer für die letzte Ölung herbeigerufen wird. Bernhardi hat in der von Oevermann sequenzanalytisch interpretierten Sequenz über das weitere Vorgehen bezüglich einer Patientin zu befinden. Entscheidungsgrundlage ist, dass die Patientin in Kürze infolge einer Sepsis nach einer Abtreibung sterben wird, wobei sie aber in völliger Unkenntnis ihrer Lage davon überzeugt ist, bald nach Hause zu 
ihrem Geliebten zurückzukehren. In konkreter Einschätzung der Situation der Patientin möchte Bernhardi die Patientin in ihrer Illusion belassen, weil er, wie Oevermann hervorhebt, auf Grundlage einer fallspezifischen Beurteilung der Situation davon überzeugt ist, dass der Patientin für eine angemessene Verarbeitung ihrer realen Situation die entsprechende Lebenszeit fehlt. Nach einem mehrmals scheiternden Vermittlungsversuch verbietet Bernhardi dem von einer Krankenschwester hinter seinem Rücken herbeigerufenen Pfarrer folgerichtig das Betreten des Krankenzimmers, weil das der Patientin unmittelbar ihre wirkliche Lage vor Augen führen würde.

Eine fallspezifische Einschätzung und Entscheidung des Arztes und Wahrung des Arbeitsbündnisses seitens des Arztes setzt hier also einen Bruch gegen Konventionen voraus, der im Falle Bernhardis auch Konsequenzen hinsichtlich seiner Karriere mit sich bringt. Das postkonventionelle Moralurteil, das gegenüber der Systemperspektive der Moralstufe 4 die Perspektive des Individuums, hier in der Wahrnehmung der Patientin in ihrer besonderen biographischen Lage, in die Urteilsfindung integriert, ermöglicht eine fallspezifische, stellvertretende Krisenlösung, ein zentrales Kriterium für professionalisiertes Handeln in Oevermann Professionalisierungstheorie.

An diesem Beispiel wird deutlich, dass professionalisiertes Handeln moraltheoretisch nicht hinreichend durch eine verlässliche Bindung an den hippokratischen Eid in der Logik der Moralstufe 4, nach der man sich auf das Wort von jemandem verlassen kann (Kegan 2008, 89), erfasst ist. Der hippokratische Eid, zu dem nach Oevermann ,[...] eben nicht nur die Lebenserhaltung im medizinisch technischen Sinne, sondern die Wahrung des Wohls der ganzen Person, also auch in ihrer seelischen Befindlichkeit“ (Oevermann 1997, 311) gehört, fordere, den Pfarrer nicht zur Patientin zu lassen und damit moralisch postkonventionell zu urteilen und zu handeln ${ }^{3}$.

3 Es ist aufschlussreich, dass ganz im Sinne der vorliegenden moraltheoretischen Untersuchung professionalisierten Handelns Oevermann in einer weiteren Fallstudie Mediziner in SS-Uniform aus professionalisierungstheoretischer Perspektive betrachtet. Hierbei handelt es sich um ein Scheitern professionalisierungsbedürftigen Handelns an einer extremen Form von System- bzw. Gruppengebundenheit. Die Perspektive des Individuums, die als eine Voraussetzung fallspezifischer, stellvertretender Krisenlösung dargestellt wurde und vollgültig erst mit dem Übergang zur postkonventionellen Ebene moralischen Urteilens in die Urteilsfindung aufgenommen werden kann, ist mit Menschenexperimenten nicht vereinbar (Oevermann 2000). 


\section{Literatur}

Fischer, C. 1996. Nachwort, in: Ch. Baudelaire, Die Blumen des Bösen, Mannheim.

Garz, D. 1996. Lawrence Kohlberg zur Einführung, Hamburg.

Goethe, J. W. 1994. Die Leiden des jungen Werthers. Die Wahlverwandtschaften. Kleine Prosa. Epen, Frankfurt am Main.

Kegan, R. 2008. Die Entwicklungsstufen des Selbst. Fortschritte und Krisen im menschlichen Leben, München.

Nickisch, R. M. G. 1991. Brief, Stuttgart.

Oevermann, U. 1996. Theoretische Skizze einer revidierten Theorie professionnalisierten Handelns, in: A. Combe, W. Helsper (Hg.), Pädagogische Professionalität. Untersuchungen zum Typus pädagogischen Handelns, Frankfurt am Main.

Oevermann, U. 1997. Literarische Verdichtung als soziologische Erkenntnisquelle: Szenische Realisierung der Strukturlogik professionalisierten ärztlichen Handelns in Arthur Schnitzlers Professor Bernhardi, in: M. Wicke, (Hg.), Konfigurationen lebensweltlicher Strukturphänomene. Soziologische Varianten phänomenologisch-hermeneutischer Welterschließung, Opladen.

Oevermann, U. 2000. Mediziner in Uniform. Professionalisierungstheoretische Deutung des Falles Münch. In: H. Kramer (Hg.), Die Gegenwart der NSVergangenheit, Berlin - Wien.

Weber, M. 1980. Wirtschaft und Gesellschaft, Tübingen. 
110 | Boris Zizek

Boris Zizek (Johannes Gutenberg-Universität Mainz)

\title{
Professionalität und Postkonventionalität. Entwicklungstheoretische und soziologische Perspektiven auf professionalisiertes Handeln
}

\begin{abstract}
The purpose of the following article is to point out the essential role of postconventional moral judgement in professionalized action. By some examples I will demonstrate that it is the precondition of completed professionalization.
\end{abstract}

Keywords. Theory and Process of Professionalization, Competence Development, Moral Post-Conventionality, Qualitative Research

Citations. Reference this paper as: Zizek, B. (2012). Professionalität und Postkonventionalität. Entwicklungstheoretische und soziologische Perspektiven auf professionalisiertes Handeln. Ethics in Progress Quarterly, Volume 3, Issue 1, pp. 97-110, available online at ethicsinprogress.org. 